\title{
A TRANSIENT SUB-EDDINGTON BLACK HOLE X-RAY BINARY CANDIDATE IN THE DUST LANES OF CENTAURUS A
}

\author{
Mark J. Burke ${ }^{1}$, Somak Raychaudhury ${ }^{1}$, Ralph P. Kraft ${ }^{2}$, Nicola J. Brassington ${ }^{3}$, Martin J. Hardcastle ${ }^{3}$, \\ Joanna L. Goodger ${ }^{3}$, Gregory R. SivakofF ${ }^{4}$, William R. Forman ${ }^{2}$, Christine Jones ${ }^{2}$, Kristin A. Woodley $^{5}$, \\ Stephen S. Murray ${ }^{2,6}$, Jouni Kainulainen ${ }^{7}$, Mark Birkinshaw $^{8}$, Judith H. Croston ${ }^{9}$, Daniel A. Evans ${ }^{2}$, \\ Marat Gilfanov ${ }^{10,11}$, Andrés Jordán ${ }^{2,12}$, Craig L. Sarazin ${ }^{13}$, Rasmus Voss ${ }^{14}$, \\ DiAna M. WORRALL ${ }^{8}$, AND ZHONGLi ZHANG ${ }^{10}$ \\ ${ }^{1}$ School of Physics and Astronomy, University of Birmingham, Edgbaston, Birmingham, B15 2TT, UK; mburke@star.sr.bham.ac.uk \\ ${ }^{2}$ Harvard-Smithsonian Center for Astrophysics, 60 Garden Street, Cambridge, MA 02138, USA \\ ${ }^{3}$ School of Physics, Astronomy, and Mathematics, University of Hertfordshire, Hatfield, AL10 9AB, UK \\ ${ }^{4}$ Department of Physics, University of Alberta, Edmonton, Alberta T6G 2E1, Canada \\ 5 Department of Physics and Astronomy, Johns Hopkins University, 3400 North Charles Street, Baltimore, MD 21218, USA \\ ${ }^{6}$ Department of Physics and Astronomy, University of British Columbia, Vancouver, BC V6T 1Z1, Canada \\ ${ }^{7}$ Max-Planck-Institute for Astronomy, Königstuhl 17, 69117 Heidelberg, Germany \\ ${ }^{8}$ HH Wills Physics Laboratory, University of Bristol, Tyndall Avenue, Bristol BS8 1TL, UK \\ ${ }^{9}$ School of Physics and Astronomy, University of Southampton, Southampton SO17 1BJ, UK \\ ${ }^{10}$ Max Planck Institut für Astrophysik, Karl-Schwarzschild-Str. 1, D-85741, Garching, Germany \\ ${ }^{11}$ Space Research Institute, Russian Academy of Sciences, Profsoyuznaya 84/32, 117997 Moscow, Russia \\ 12 Departamento de Astronomía y Astrofísica, Pontificia Universidad Católica de Chile, 7820436 Macul, Santiago, Chile \\ ${ }^{13}$ Department of Astronomy, University of Virginia, P.O. Box 400325, Charlottesville, VA 22904-4325, USA \\ ${ }^{14}$ Department of Astrophysics/IMAPP, Radboud, University Nijmegen, P.O. Box 9010, NL-6500 GL Nijmegen, The Netherlands \\ Received 2011 November 29; accepted 2012 February 9; published 2012 March 28
}

\begin{abstract}
We report the discovery of a bright X-ray transient CXOU J132527.6-430023 in the nearby early-type galaxy NGC 5128. The source was first detected over the course of five Chandra observations in 2007, reaching an unabsorbed outburst luminosity of (1-2) $\times 10^{38} \mathrm{erg} \mathrm{s}^{-1}$ in the $0.5-7.0 \mathrm{keV}$ band before returning to quiescence. Such luminosities are possible for both stellar-mass black hole and neutron star (NS) X-ray binary transients. Here, we attempt to characterize the nature of the compact object. No counterpart has been detected in the optical or radio sky, but the proximity of the source to the dust lanes allows for the possibility of an obscured companion. The brightness of the source after a $>100$-fold increase in X-ray flux makes it either the first confirmed transient non-ultraluminous X-ray black hole system in outburst to be subject to detailed spectral modeling outside the Local Group, or a bright $\left(>10^{38} \mathrm{erg} \mathrm{s}^{-1}\right)$ transient NS X-ray binary, which are very rare. Such a large increase in flux would appear to lend weight to the view that this is a black hole transient. X-ray spectral fitting of an absorbed power law yielded unphysical photon indices, while the parameters of the best-fit absorbed disk blackbody model are typical of an accreting $\sim 10 M_{\odot}$ black hole in the thermally dominant state.
\end{abstract}

Key words: galaxies: elliptical and lenticular, cD - galaxies: individual (Centaurus A, NGC 5128) - X-rays: binaries - X-rays: galaxies

Online-only material: color figures

\section{INTRODUCTION}

The majority of confirmed black hole low- and high-mass $\mathrm{X}$-ray binary (BH LMXB, HMXB) systems are transient sources that experience outbursts lasting from a few weeks to a matter of years. These outbursts are characterized by an increase in $\mathrm{X}$-ray luminosity by many orders of magnitude from a quiescent state of $L_{X} \sim 10^{30}-10^{34} \mathrm{erg} \mathrm{s}^{-1}$, recurring on timescales of months to decades (for a review see Remillard \& McClintock 2006). The recurrent behavior of the persistent luminosity, characterized by a rapid rise and exponential decay, is thought to be the result of accretion disk instability (Dubus et al. 2001). Research into Galactic BH LMXBs has revealed three common spectral states between which sources are observed to transit; a thermal state dominated by radiation originating in the inner regions of a geometrically thin, optically thick accretion disk (Shakura \& Sunyaev 1973), a hard state dominated by a power law with photon index $\Gamma \sim 1.7$, and a steep power-law state $\Gamma \sim 2.5$ extending to $\mathrm{MeV}$ energies where a significant thermal component is also present. The similarity of these states to the spectra of neutron star (NS) LMXBs means that it is often impossible to fully distinguish between bright NS and $\mathrm{BH}$ LMXBs on the basis of luminosity and spectra alone. However, a distinction can be drawn between the thermal states, which are typically much harder in NS systems than for BH sources. This is in agreement with the proposal that "ultrasoft" spectra are a characteristic of BH sources (White \& Marshall 1984). NS systems, as a result of the presence of the physical surface of the compact object, experience behavior in the form of bursting, for lower-luminosity (Atoll) sources such as $4 \mathrm{U}$ 1636-536, and flaring, for more luminous ( $L_{X} \sim 10^{38} \mathrm{erg} \mathrm{s}^{-1}$, Z-track) systems such as Sco X-1.

Until recently, only two transient NS LMXBs with $L_{X} \sim$ $L_{\text {Edd }}$ were known, Cir X-1 and XTE J1806-246. Cir X-1 is a peculiar NS XB with atypical spectral and timing behavior and was once thought to possess a BH primary, an idea which was confounded by the detection of Type-I X-ray bursts (Tennant et al. 1986; Linares et al. 2010). The transient nature of Cir X-1 
is also unusual in that it is periodic with a period of 16.6 days and therefore thought to be the result of the eccentric orbit of the donor star (Murdin et al. 1980), rather than accretion disk instability. The peak luminosity of XTE J1806-246, reported as $L_{X} \sim 1.5 \times 10^{38} \mathrm{erg} \mathrm{s}^{-1}$ (Wijnands \& van der Klis 1999), is based on a poor distance estimate $(8 \mathrm{kpc}$, based on a sky position coincident with the Galactic bulge). The recent discovery of the transient NS LMXB XTE J1701-462 (Homan et al. 2007), which experienced the full range of spectral and timing behavior observed in all other Galactic NS LMXBs (Lin et al. 2009; Sanna et al. 2010) during a $\sim 600$ day outburst, demonstrates both that NS transients can be as luminous as some of their BH cousins, and that the short-term variability is fundamentally linked to the accretion rate of the system.

The Chandra and XMM-Newton X-ray observatories have made possible the detailed study of extragalactic X-ray binary (XB) sources during the last decade. Within the Local Group, Williams et al. (2006) cataloged the properties of 45 transient $\mathrm{XBs}$ in $\mathrm{M} \mathrm{31}$, and found that most exist around the central bulge of the galaxy, suggesting that they are part of an older stellar population and therefore more likely to be LMXBs. More detailed studies of brighter transient sources (Trudolyubov et al. 2006) and the overall XB population (Stiele et al. 2011) show many soft sources, with absorbed disk blackbody temperatures $k T_{\text {in }}<1 \mathrm{keV}$, and $<0.1 \mathrm{keV}$ in the case of super-soft sources (SSS) that show no emission above $1 \mathrm{keV}$. Such low values of $k T_{\text {in }}$ are inconsistent with spectral fitting results for Galactic NS LMXB (e.g., Revnivtsev \& Gilfanov 2006) and are thus indicative of $\mathrm{BH}$ systems in the thermal state, while SSS are thought to be accreting white dwarfs (van den Heuvel et al. 1992; Kahabka \& van den Heuvel 1997). It has been shown that the M 31 X-ray transients are found in three distinct spectral groups at their peak luminosity (Voss et al. 2008), categorized as very soft, soft, and hard, half of all the transients being hard. The ChASeM33 project, consisting of $7 \times 200 \mathrm{ks}$ Chandra exposures of $\mathrm{M} 33$, allowed source detection to a depth of $5 \times 10^{34} \mathrm{erg} \mathrm{s}^{-1}$, and seven transient sources have been discovered, albeit only two with $L_{X}>10^{37} \mathrm{erg} \mathrm{s}^{-1}$ (Williams et al. 2008).

Outside the Local Group, in the elliptical galaxies NGC 3379 and NGC 4278 (Brassington et al. 2008, 2009), five and three sources respectively have been classified as transient candidates (TCs). Three other sources in each of these galaxies have been identified as possible transient candidates (PTCs). TCs and PTCs correspond to observed changes in luminosity by factors of $>10$ and $>5$ respectively, and we note that some Galactic sources such as 4U 1705-44 (Homan et al. 2009) and 4U 0513-40 (Maccarone et al. 2010) are not transients but do show variations significantly $>10$. All of these sources have hardness ratios and $\mathrm{X}$-ray colors that are consistent with Galactic LMXBs. Followup spectral analysis on the bright, seemingly persistent sources in these galaxies (Brassington et al. 2010; Fabbiano et al. 2010) yielded $L_{X} \sim 10^{38}-10^{39} \mathrm{erg} \mathrm{s}^{-1}$, with spectral fitting parameters congruent with known BH systems; the strongest BH binary candidate had maximum and minimum unabsorbed luminosities of $1.2 \times 10^{39} \mathrm{erg} \mathrm{s}^{-1}$ and $4.1 \times 10^{38} \mathrm{erg} \mathrm{s}^{-1}$.

For observational reasons, the XBs outside the Local Group that have been the most intensely studied are the Ultraluminous $\mathrm{X}$-ray sources (ULXs), which possess outburst luminosities in excess of $10^{39} \mathrm{erg} \mathrm{s}^{-1}$, together with other bright sources with luminosities typically $>2-3 L_{\text {Edd }}$ for an accreting NS. These sources represent a comparatively small fraction of XBs in the Local Group, with the solitary ULXs found in M 31 and M
33 being the only constituents of their class (Kaur et al. 2012; Dubus \& Rutledge 2002). Therefore, there exists an asymmetry between the study of XBs inside and outside the Local Group.

\subsection{NGC 5128}

Centaurus A (NGC 5128, Cen A) is the nearest optically luminous large early-type galaxy, situated at a distance of 3.7 Mpc (Ferrarese et al. 2007), with $M_{B}=-21.1$. A small late-type galaxy is currently merging with Cen A, however, the galaxies remain poorly mixed (Quillen et al. 2006). The merger has resulted in the presence of vast dust lanes that obscure many of the central regions at optical and soft X-ray wavelengths (Graham 1979).

Six $100 \mathrm{ks}$ Chandra observations were taken as part of the Cen A Very Large Project (VLP) spanning the course of two months (Jordán et al. 2007). These observations have allowed unprecedented insight into the X-ray jets (Hardcastle et al. 2007; Worrall et al. 2008; Goodger et al. 2010), radio-lobe shock (Croston et al. 2009), extended gaseous emission (Kraft et al. 2008), and work on the XB, particularly in relation to globular clusters (Voss et al. 2009, see also Voss \& Gilfanov 2006 and Woodley et al. 2008 using pre-VLP data).

Two transients in the field of Cen A have previously been studied. Sivakoff et al. (2008) discovered a previously undetected transient using VLP observations, CXOU J132518.2-430304. With outburst luminosities in excess of $10^{39} \mathrm{erg} \mathrm{s}^{-1}$, it is by definition a ULX. CXOU J132518.2-430304 underwent a >770fold increase in luminosity between quiescent and active states, with an outburst duration of $>70$ days. Spectral analysis coupled with Hubble Space Telescope (HST) observations strongly suggest that the source is a BH LMXB that transitions from the steep power-law state to the thermally dominant state over the course of the VLP observations. Prior to this, Kraft et al. (2001) had detected a transient ULX, CXOU J132519.9-43031, $\left(L_{X} \sim 10^{39} \mathrm{erg} \mathrm{s}^{-1}\right)$ within $\sim 6^{\prime \prime}$ of a source detected by ROSAT over the course of 10 days in 1995. Spectral fitting suggested the source was in a steep power-law state (see also Ghosh et al. 2006).

In this paper, we present the first detailed analysis of a sub-Eddington X-ray transient at Mpc distances, CXOU J132527.6-430023, whose flux rose by more than a factor of 100 from quiescence to outburst and reached peak unabsorbed luminosities of $\sim 2 \times 10^{38} \mathrm{erg} \mathrm{s}^{-1}(0.5-7.0 \mathrm{keV})$ before fading back to a quiescent state by MJD 54250 (Table 1). We present evidence that this source is a BH LMXB and compare it to LMXB systems observed both inside and outside the Local Group. This source is clearly a bridge between more typical Galactic and extragalactic XBs. Since Cen A is nearby, this is the only sub- (or $\sim$ ) Eddington transient where we can perform detailed spectral fitting and attempt to classify the compact as a $\mathrm{BH}$ or an NS.

\section{DATA PREPARATION AND ANALYSIS}

\subsection{Preliminary Work}

An overview of all Chandra Advanced CCD Imaging Spectrometer (ACIS) observations of Cen A can be found in Table 1. All of the $5 \mathrm{ks}$ and $50 \mathrm{ks}$ observations of Cen A were taken with the ACIS as part of the HRC Guaranteed Observation Time program (PI: Murray).

All the data were reprocessed using CIAO 4.3 and HEASOFT 6.11 with CALDB 4.4.2. All observations were first co-aligned to a precision of $<0$ '. 2 based on the positions of the point sources 

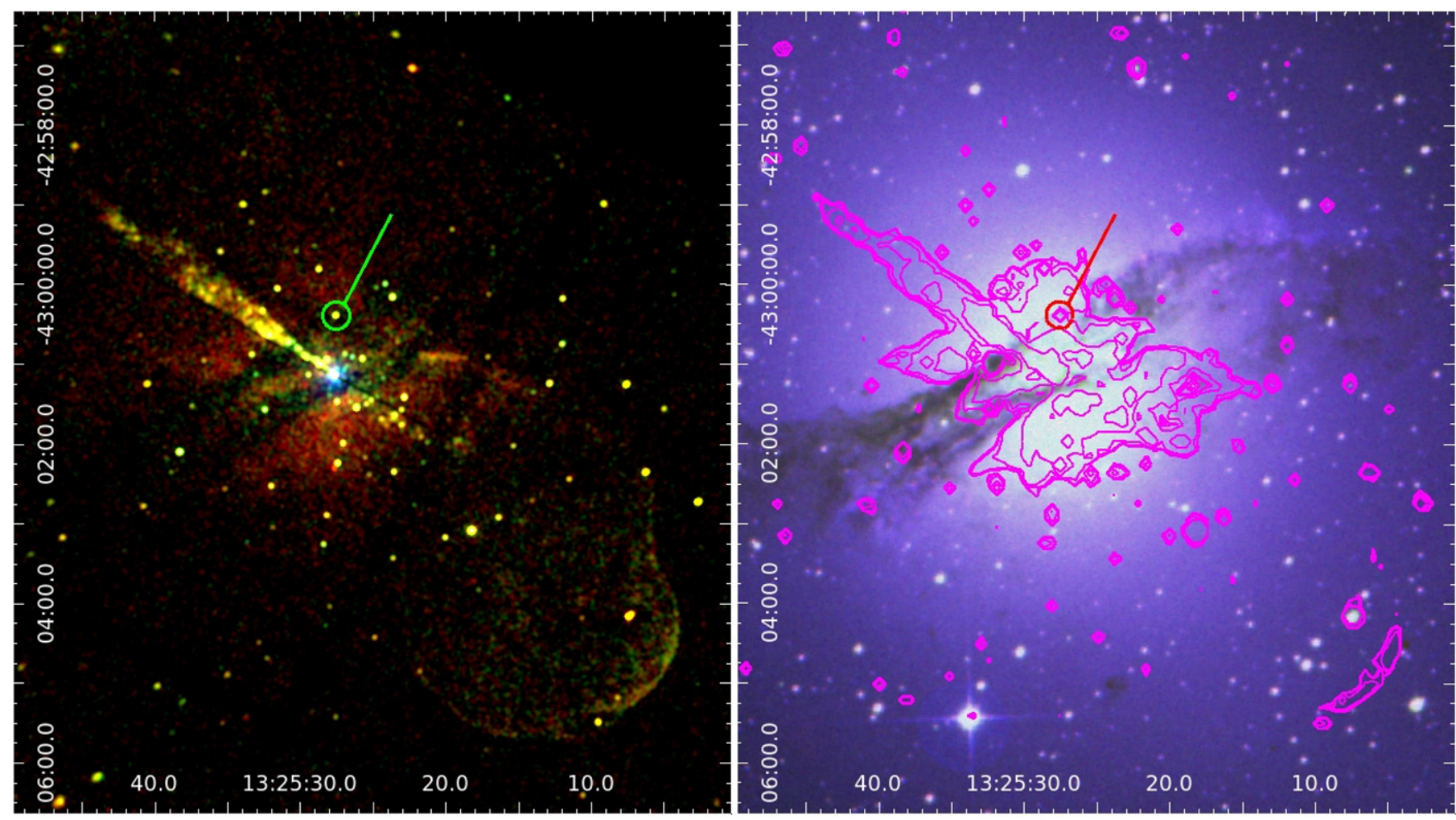

Figure 1. Location of CXOU J132527.6-430023 within Cen A. Left: a Gaussian-smoothed logarithmic scale 0.5-1.3 keV (red), 1.3-2 keV (green), and 2-7 keV (blue) image of ObsID 7797. Right: X-ray 0.5-7 keV contours of the brighter central regions of Cen A (Magenta) overlaid on Deep Sky Survey combined $R-, I-$, and $B$-band images of Cen A.

(A color version of this figure is available in the online journal.)

Table 1

ACIS-I/S Observations of Transient in Cen A

\begin{tabular}{|c|c|c|c|c|}
\hline ObsID & Instrument & $\begin{array}{c}\text { Exposure } \\
(\mathrm{ks})\end{array}$ & $\begin{array}{c}\text { Date } \\
\text { MJD (yyyy-mm-dd) }\end{array}$ & Net Counts \\
\hline 316 & ACIS-I & 36.18 & $51517(1999-12-05)$ & $<2.7$ \\
\hline 962 & ACIS-I & 36.97 & $51681(2000-05-17)$ & $<4.3$ \\
\hline 2978 & ACIS-S & 45.18 & $52520(2002-09-03)$ & $<4.3$ \\
\hline 3965 & ACIS-S & 50.17 & $52896(2003-09-14)$ & $<4.0$ \\
\hline 7797 & ACIS-I & 98.17 & $54181(2007-03-22)$ & $336 \pm 32$ \\
\hline 7798 & ACIS-I & 92.04 & $54186(2007-03-27)$ & $364 \pm 34$ \\
\hline 7799 & ACIS-I & 96.04 & $54189(2007-03-30)$ & $375 \pm 35$ \\
\hline 7800 & ACIS-I & 92.05 & $54207(2007-04-17)$ & $284 \pm 31$ \\
\hline 8489 & ACIS-I & 95.18 & $54228(2007-05-08)$ & $16_{-7}^{+9}$ \\
\hline 8490 & ACIS-I & 95.68 & $54250(2007-05-30)$ & $<7.3$ \\
\hline 10723 & ACIS-I & 5.15 & 54835 (2009-01-04) & $<2.6$ \\
\hline 10724 & ACIS-I & 5.17 & $54895(2009-03-05)$ & $<2.6$ \\
\hline 10725 & ACIS-I & 5.04 & $54947(2009-04-26)$ & $<2.6$ \\
\hline 10726 & ACIS-I & 5.15 & $55003(2009-06-21)$ & $<4.0$ \\
\hline 10722 & ACIS-S & 50.04 & $55082(2009-09-08)$ & $<2.3$ \\
\hline 11846 & ACIS-I & 4.75 & $55312(2010-04-26)$ & $<3.9$ \\
\hline 11847 & ACIS-I & 5.05 & $55455(2010-09-16)$ & $<2.6$ \\
\hline 12155 & ACIS-I & 5.05 & $55552(2010-12-22)$ & $<2.6$ \\
\hline 12156 & ACIS-I & 5.06 & $55734(2011-06-22)$ & $\cdots$ \\
\hline
\end{tabular}

Notes. ${ }^{\text {a }}$ For observations where the source was not detected, the net counts column represents the $90 \%$ upper limit placed on the net counts of the source in the $0.5-2.0 \mathrm{keV}$ band. For the detections (bold), $90 \%$ confidence regions are shown. The position of the source is coincident with the ACIS readout streak in ObsID 12156 and no upper limit was calculated in this instance.

detected using wavdetect and then aligned to the positions of confirmed Cen A globular clusters. This procedure will be fully described in a later paper. CXOU J132527.6-430023 was detected in Chandra ObsIDs 7797, 7798, 7799, 7800, and 8489.

We measured the position of CXOU J132527.6-430023 as $\alpha=13^{\mathrm{h}} 25^{\mathrm{m}} 27^{\mathrm{s}} .58, \delta=-43^{\circ} 00^{\prime} 23^{\prime \prime} .3$ (J2000) with $<0^{\prime \prime} .2$ uncertainty (Figure 1). The $0.5-2.0 \mathrm{keV}$ light curve across the $100 \mathrm{ks}$ observations was generated using the ciao tool aprates. The source flux was calculated based on a circular region of radius $r_{p 2}$ equal to $90 \%$ of the $2 \mathrm{keV}$ point-source function (PSF) centered at the off-axis position of the source, and the background flux from an annulus extending from $r_{p 2}$ to $3 r_{p 2}$. No other sources are seen overlapping with these source regions, and no other $r_{p 2}$ source regions encroach on the background annuli. In Figure 2, we present light curves in the $0.5-2.0 \mathrm{keV}$ band, with the VLP (outburst) light curve shown in the bottom panel, while the top panel shows the overall ACIS light curve of the source. We restricted ourselves to this low-energy range due to the proximity of the source to the AGN. For the six $100 \mathrm{ks}$ observations, the mean photon energies per effective exposure of each $0.5-2.0 \mathrm{keV}$ event were first calculated using eff 2 evt on both the source and the background regions, which are then used as aprates input parameters. For the upper limits, the exposure times of the source and background regions were also used. aprates then performed a Bayesian analysis and returned the net $0.5-2.0 \mathrm{keV}$ flux with a $90 \%$ confidence interval or count-rate upper limit. A combined upper limit was calculated for the $7 \times 5 \mathrm{ks}$ observations and individual upper limits were obtained for each observation of exposure $>30 \mathrm{ks}$. The upper limits were then converted to $0.5-2.0 \mathrm{keV}$ fluxes, uncorrected for absorption, using the Chandra X-Ray Center program PIMMS, assuming an absorbed power law spectrum with $\Gamma=2.0$ and $N_{\mathrm{H}}=0.084 \times 10^{22} \mathrm{~cm}^{-2}$ (i.e., a relatively steep spectrum for a quiescent LMXB in front of the dust lane). 


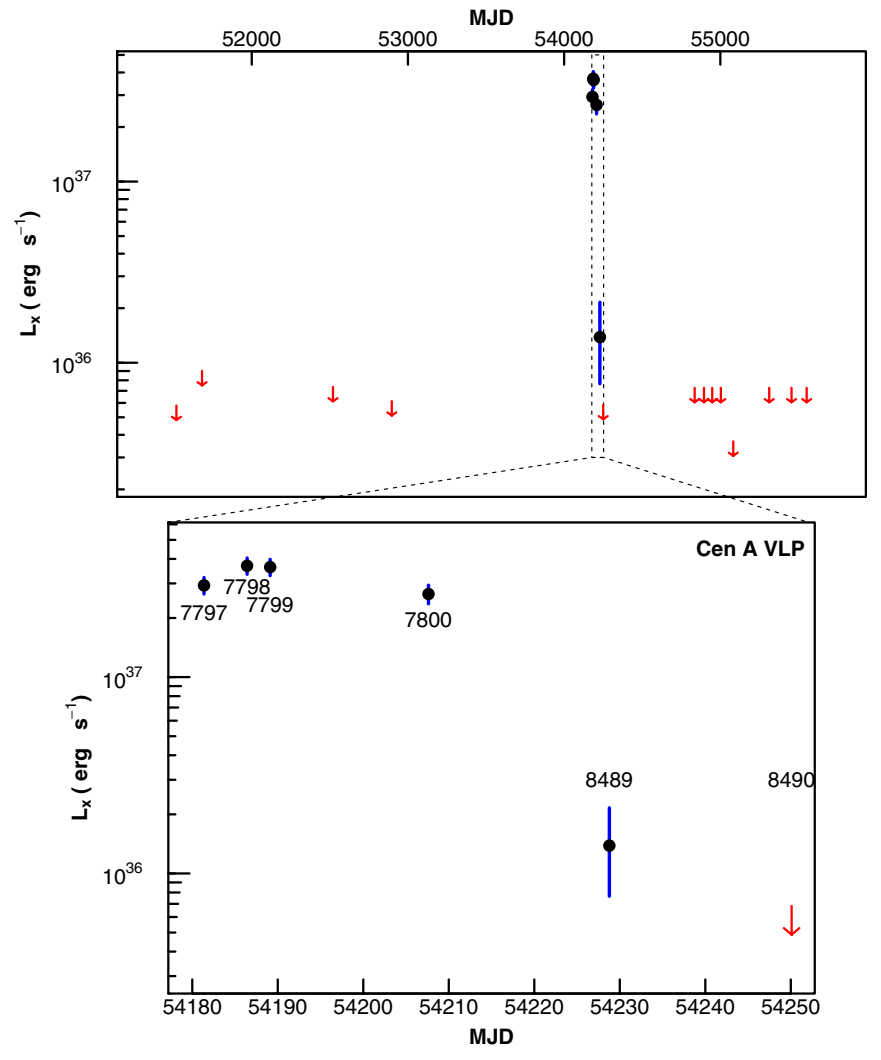

Figure 2. X-ray light curve $(0.5-2 \mathrm{keV})$, uncorrected for absorption. Top: luminosity is displayed with $90 \%$ confidence intervals (blue error bars) and with $90 \%$ upper limits from other ACIS observations (red arrows). A combined upper limit was obtained from the $5 \mathrm{ks}$ observations (the seven arrows to the right). Bottom: light curve of VLP observations.

(A color version of this figure is available in the online journal.)

The source position is obscured by the ACIS readout streak in ObsID 12156, no upper limit was obtained in this case. With aprates, the combined upper limit on count rate was calculated by inputting the summation of counts, areas, and exposure times of the source and background regions. Measured fluxes and upper limits were then converted into luminosity using a distance of $3.7 \mathrm{Mpc}$. We recognize that a more recent measurement has produced a distance to Cen A of $3.8 \pm 0.1 \mathrm{Mpc}$ (Harris et al. 2010), but we have used a distance of 3.7 Mpc in calculations throughout this paper for the sake of consistency with earlier work. This said, the uncertainty of the distance measurement is much less than the uncertainties associated with the calibration and spectral fitting process, which therefore have a greater effect on our luminosity measurement. ObsID 10722 is our lowest luminosity upper limit, $L_{X}>3 \times 10^{35} \mathrm{erg} \mathrm{s}^{-1}$.
Several factors make CXOU J132527.6-430023 the best transient $\mathrm{BH}$ candidate in Cen A to study in the $\sim 10^{38} \mathrm{erg} \mathrm{s}^{-1}$ regime. It is clear that it is observed in both outburst and quiescent states during the VLP observations. Basic observational criteria regarding the source are satisfied; the source is always in the field of view, far from the ACIS chip edges and has the required counts to perform meaningful spectral analysis. Our work, currently in preparation, shows that no other source, observed to be transient in Cen A during the VLP, is as bright in as many observations while also undergoing such a dramatic change in the mean flux (Figure 2), as CXOU J132527.6-430023.

Before the extraction of spectra, we investigated the intraobservation variability by extracting a count-rate light curve of each observation, to test if the source was active throughout a given observation, or was prone to more discreet behavior. In Figure 3, we present the intra-observation $0.5-2.0 \mathrm{keV}$ light curve, with count rate grouped to $10 \mathrm{ks}$ bins. The mean intensity had substantially diminished by the time of ObsID 8489 , approximately 20 days after the previous observation. A simple $\chi^{2}$ test showed no significant variations from the best-fit straight line for any of the $100 \mathrm{ks}$ Chandra pointings binned to $10 \mathrm{ks}$.

\subsection{Possible Counterparts}

We investigated the possibility of detecting a counterpart to CXOU J132527.6-430023 at other wavelengths. We examined $B$ - and $R$-band images from recent observations (Harris et al. 2012) with the Walter Baade Telescope and the Inamori Magellan Areal Camera (IMACS) and found no point sources coincident with the position of the transient. Further investigation using an HST WFPC2 observation, U4100108M ( F814W) showed no optical counterpart. However, the position is coincident with the edge of the dust lane. This being the case, any attempt to perform optical photometry on the source position will be inconclusive. The nearest globular cluster that has been spectroscopically confirmed as belonging to Cen $\mathrm{A}$ is $~ 30^{\prime \prime}$ away from CXOU J132527.6-430023 (Woodley et al. 2008). However, no clusters have been detected within the radial distance of the transient from the galaxy center, as a result of the high stellar luminosity within $\sim 1.5$ of the Cen A nucleus, in tandem with the heavy obscuration by the dust lanes.

A 2007 June Very Large Array 8.4 GHz observation using the A configuration, obtained just one week after the final VLP observation, places a $3 \sigma$ upper limit of $0.13 \mathrm{mJy}$ on the radio emission at this position. Furthermore, it has been confirmed that no radio counterpart has been detected in any other VLP observation (Goodger et al. 2010). Galactic transient XBs frequently show evidence of jets during outburst (see Fender 2005 for review), and have a clear relationship between the strength of the outburst X-ray luminosity and the flux density

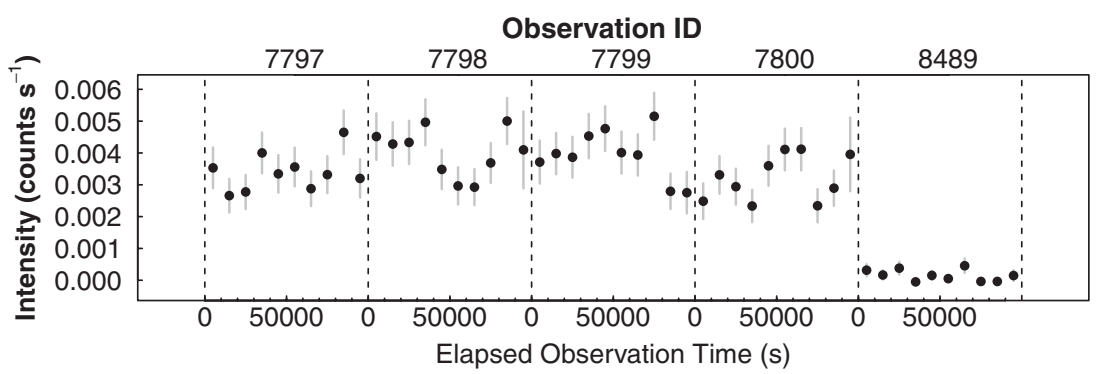

Figure 3. Intra-observation $0.5-2.0 \mathrm{keV}$ light curves of the net intensity with $10 \mathrm{ks}$ binning within the VLP observations. For the ObsID dates, see Table 1. 
Table 2

Best-fit Parameter Values: phabs $\times$ diskbb Model

\begin{tabular}{cccccc}
\hline \hline ObsID & $\begin{array}{c}N_{\mathrm{H}} \\
\left(10^{22} \mathrm{~cm}^{-2}\right)\end{array}$ & $\begin{array}{c}\text { Flux }^{\mathrm{a}, \mathrm{b}} \\
\left(10^{-14 \mathrm{~d}}\right)\end{array}$ & $\begin{array}{c}k T_{\text {in }} \\
(\mathrm{keV})\end{array}$ & $\chi^{2} / \mathrm{dof}$ & $\begin{array}{c}L_{\mathrm{x}}^{\mathrm{a}, \mathrm{b}, \mathrm{c}} \\
\left(10^{38} \mathrm{erg} \mathrm{s}^{-1}\right)\end{array}$ \\
\hline 7797 & $0.36_{-0.19}^{+0.22}$ & $7.69_{-1.44}^{+2.04}$ & $0.65_{-0.10}^{+0.11}$ & $15.85 / 16$ & $1.26_{-0.24}^{+0.33}$ \\
7798 & $0.43_{-0.18}^{+0.22}$ & $7.93_{-1.49}^{+2.22}$ & $0.61_{-0.10}^{+0.12}$ & $15.18 / 19$ & $1.30_{-0.25}^{+0.36}$ \\
7799 & $0.83_{-0.23}^{+0.29}$ & $12.12_{-2.97}^{+5.05}$ & $0.51_{-0.08}^{+0.09}$ & $11.83 / 19$ & $1.99_{-0.49}^{+0.82}$ \\
7800 & $0.33_{-0.22}^{+0.27}$ & $4.30_{-1.07}^{+1.92}$ & $0.53_{-0.11}^{+0.13}$ & $11.75 / 14$ & $0.70_{-0.17}^{+0.32}$ \\
\hline
\end{tabular}

Notes.

a Spectral fits to the $0.5-7.0 \mathrm{keV}$ band.

${ }^{\mathrm{b}}$ Unabsorbed, from the XSPEC cflux parameter.

${ }^{\mathrm{c}} L_{X}$ based on a distance of $3.7 \mathrm{Mpc}$.

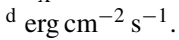

of the radio emission. Sources typically possess a flat radio spectrum, with flux densities $\sim 1-1000 \mathrm{mJy}$ when scaled to $1 \mathrm{kpc}$. Scaling this to the distance to Cen A suggests that the expected flux density of a typical counterpart would lie in the range $0.07 \mathrm{nJy}$ to $0.07 \mu \mathrm{Jy}$, far smaller than our upper limit. Cyg $\mathrm{X}-3$ is an HMXB where the evidence of a $\mathrm{BH}$ is fairly strong (Shrader et al. 2010) and has been seen to emit strong radio flares of up to $20 \mathrm{Jy}$ from an assumed distance of $9 \mathrm{kpc}$ (Corbel et al. 2012); at the distance of Cen A such an outburst would be detected above our upper limit. Therefore, a radio detection of an XB counterpart in Cen A would point toward a high-mass companion.

\subsection{Spectral Fitting}

Spectra and responses in the $0.5-7.0 \mathrm{keV}$ range for each of the five observations where the source is detected were generated, based on circular apertures of radius $r_{p 7}$ equal to $90 \%$ of the $7 \mathrm{keV}$ PSF size at the off-axis source position. Background spectra were taken from an annulus $2 r_{p 7}$ to $4 r_{p 7}$, minus two overlapping circular $r_{p 2}$ source regions corresponding to two faint sources that were only detected in the merged $600 \mathrm{ks}$ data set. In the four bright observations, 7797-7800, the spectra were grouped to a minimum of 20 counts channel ${ }^{-1}$ to allow use of the $\chi^{2}$-statistic, whereas the spectrum from observation 8489 contained too few counts for grouping and fitting. All fitting was performed using XSPEC 12.7.0 (Arnaud 1996).

While the large range in flux from the source negates the possibility of it being a background object, such as an active galactic nucleus (AGN), it is prudent to check that the source is not a foreground flare star. We tested this by attempting to fit an absorbed thermal plasma model (phabs $\times$ apec). For the default settings (abundance fixed at 1.0) fit statistics for the spectra from ObsId 7797 to ObsId 7800 were $\chi^{2} /$ dof $=56.52 / 17,58.1 / 20,102.4 / 20$, and $44.1 / 15$ $\left(\chi_{v}^{2}>2\right)$, respectively. Allowing the abundance to vary led to good fits, $\chi_{v}^{2} \sim 0.8-1.4$, but the abundance tended toward zero, approximating a pure bremsstrahlung spectrum. Coupled with an apparent lack of optical counterpart it is reasonable to discount the possibility of CXOU J132527.6-430023 being a foreground object.

We followed the spectral fitting prescription of Brassington et al. (2010), who developed a consistent approach to fitting the spectra of luminous LMXBs in NGC 3379. Absorbed single-component models, starting with a simple power law, are fitted first, and the nature of these fits informs a decision on how to proceed to further fitting. Each observation was individually fitted in XSPEC with a phabs $\times$ powerlaw model,

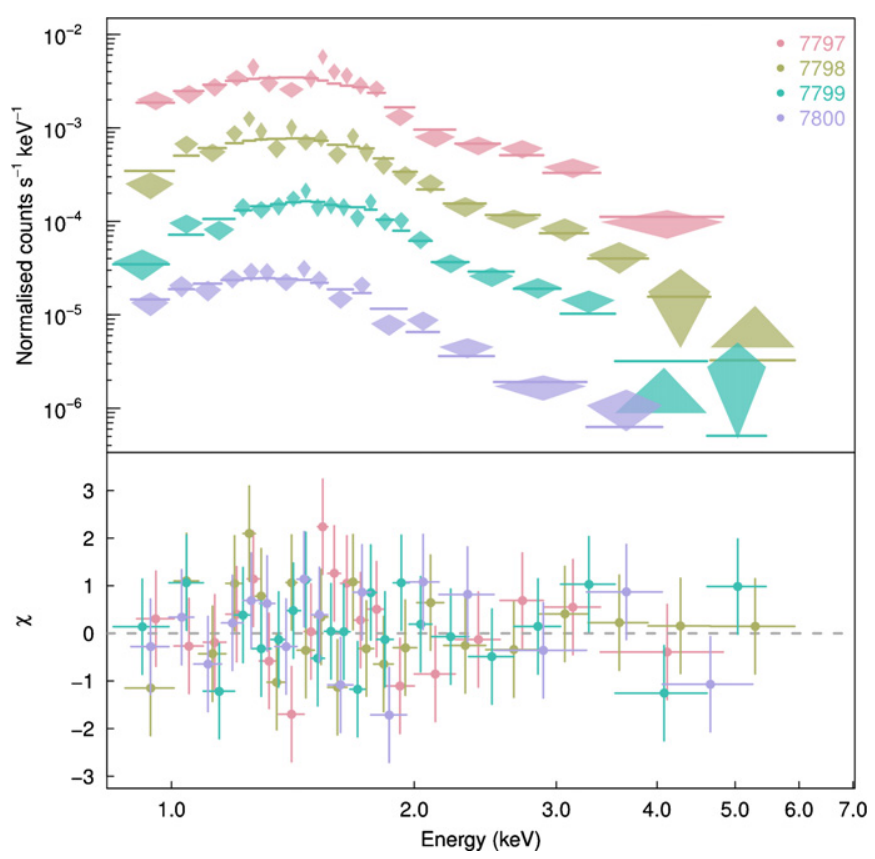

Figure 4. Spectra of the four bright VLP observations of CXOU J132527.6-430023. Spectra subsequent to 7797 are offset in $y$ by a factor $\times 5^{-n}$, where $n=1$ for $7798, n=2$ for 7799 , etc. Spectra values and associated errors are represented by diamonds while the absorbed disk blackbody model is represented by horizontal lines. Triangles show instances where the uncertainty was greater than the rate in the channel.

(A color version of this figure is available in the online journal.)

all parameters of which were left free. Successful fits were achieved for the four observations where the source was bright, with the largest $\chi_{v}^{2} \sim 1.1$. The fitting yielded quite high values for the photon index, $\Gamma \sim 3.7-4.7$, while the absorption column was above the Galactic value of $N_{\mathrm{H}}=0.084 \times 10^{22} \mathrm{~cm}^{-2}$ (Dickey \& Lockman 1990), with $N_{\mathrm{H}} \sim 0.9-1.7 \times 10^{22} \mathrm{~cm}^{-2}$, also larger than the inferred range of the dust lanes and in the $2^{\prime \prime}$ vicinity of the source (Section 2.2). The results of the simulations of Brassington et al. (2010) suggest that XSPEC absorbed power-law fits on LMXB will increase the value of $N_{\mathrm{H}}$ as a compensation for instances where there is a lack of a required thermal component. It was therefore appropriate to fit an absorbed multicolored disk blackbody model ( $p h a b s \times$ $d i s k b b$ in XSPEC) and contrast the two sets of fit results.

The absorbed disk blackbody was the best-fit model for all four of the bright spectra (Table 2), with $\chi_{v}^{2}<1$. The addition of a second additive component, such as a power law, cut-off power law, or compTT proved unnecessary, as their normalizations tended toward zero. Table 2 shows that the $N_{\mathrm{H}}$ in the phabs $\times$ diskbb fits is significantly less than for the phabs $\times$ powerlaw model, above the Galactic value and, for three of the spectra, consistent with the range of $N_{\mathrm{H}} \sim$ $(0.11-0.66) \times 10^{22} \mathrm{~cm}^{-2}$ inferred from Schreier et al. (1996). However, this is still less than our calculated average value $\left(N_{\mathrm{H}} \sim 0.6 \times 10^{22} \mathrm{~cm}^{-2}\right)$ over a $2^{\prime \prime}$ region from a $K$-band optical depth map. These results suggest that the source exhibited minor spectral evolution across the four bright observations (Figure 4).

In order to test whether the apparent difference in absorption between ObsID 7799 and the other spectra was a real effect or the result of stochastic spectral variation, a joint fit using the spectra from ObsIDs 7797, 7798, and 7800 was performed, achieving $\chi_{v}^{2} \sim 48.5 / 53$. This produced more constrained parameter values of $N_{\mathrm{H}}=0.38 \pm 0.12 \times 10^{22} \mathrm{~cm}^{-2}$ and 


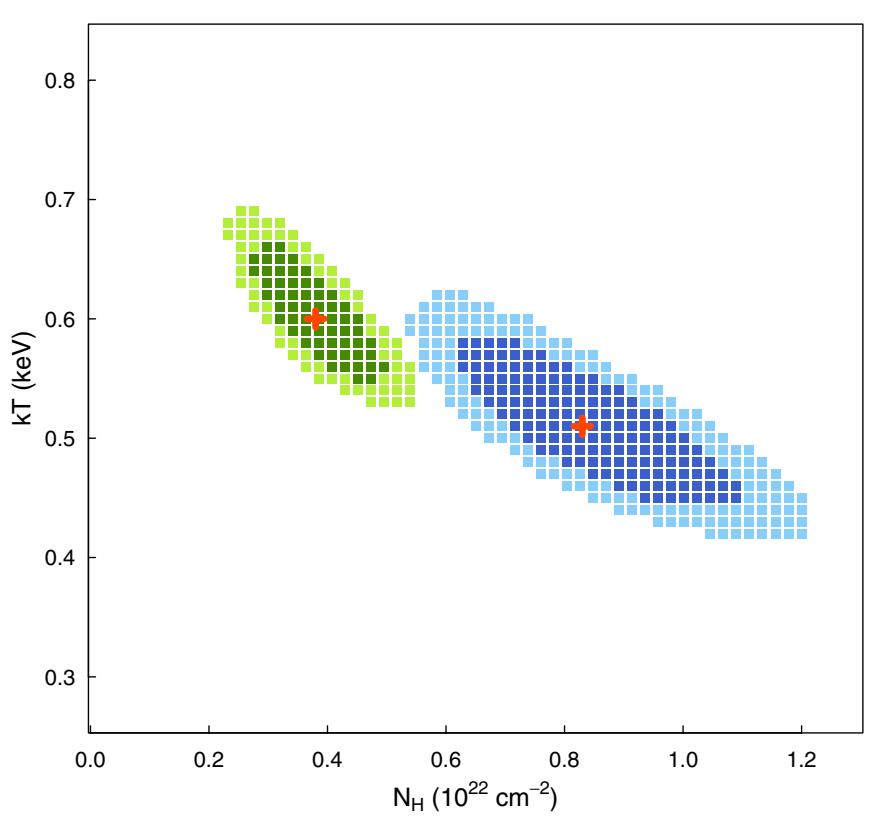

Figure 5. 90\% and 68\% confidence regions with best-fit values (red crosses) Left: from joint spectral fit to ObsIDs 7797, 7798, and 7800. Right: from spectral fit of ObsID 7799 .

(A color version of this figure is available in the online journal.)

$k T_{\text {in }}=0.60 \pm 0.05 \mathrm{keV}$. The three observations would have luminosities of $1.28 \times 10^{38} \mathrm{erg} \mathrm{s}^{-1}, 1.22 \times 10^{38} \mathrm{erg} \mathrm{s}^{-1}$, and $7.2 \times 10^{37} \mathrm{erg} \mathrm{s}^{-1}$, respectively. We investigated the spectral fitting behavior in the $k T_{\text {in }}-N_{\mathrm{H}}$ plane across a $50 \times 50$ grid of the parameter space (Figure 5). These results suggest that we can reject consistency of $N_{\mathrm{H}}$ at the $2 \sigma$ level between the joint fit and the fit to ObsID 7799. This does suggest that there is a genuine change in absorption local to the source during $\mathrm{Ob}$ sID 7799. A change in local absorption has previously been established in other BH systems. Oosterbroek et al. (1997) simulated spectra of various model parameters in an attempt to infer which change in parameter space could reproduce the observed color-color diagrams of GS $2023+338$. They concluded that a large increase in the local absorption was the best explanation of the observed color-color tracks, and speculated that the system could be inclined enough for the absorbing material to originate in the edge of the accretion disk. Such a change in absorption has also been reported from an extragalactic BH source, XMMU 122939.7+07533 (Maccarone et al. 2007). This was at first attributed to a warping of the accretion disk (Shih et al. 2010), but the presence of strong, broad O III lines (Zepf et al. 2008) suggests that a strong disk wind varies the amount of absorbing material along the line of sight.

\section{DISCUSSION}

In this section, we compare the properties of the transient source CXOU J132527.6-430023 to other XBs, both Galactic and extragalactic in origin, in the hope of finding wellstudied analogues. The outburst duration is between $\sim 50$ and $\sim 1500$ days. This is consistent with outbursts observed in many Galactic sources, which are active on timescales of months to years-much longer than so-called fast X-ray transients, for which the outburst duration is less than a day (Heise $\&$ in't Zand 2005). The peak unabsorbed luminosity of $\sim 2 \times 10^{38} \mathrm{erg} \mathrm{s}^{-1}$ is close to the Eddington luminosity of an accreting $1.4 M_{\odot} \mathrm{NS}$; however, it is possible that the source reached its peak luminos- ity prior to first being detected. The nature of the compact object is ambiguous based solely on the luminosity.

Samples of luminous LMXBs from NGC 3379 and NGC 4278 have been used to give an indication of $\mathrm{BH}$ mass (Brassington et al. 2010; Fabbiano et al. 2010) based on the $L_{X}-k T_{\text {in }}$ relation of Gierliński \& Done (2004). In agreement with the broad trend shown by these more luminous sources, the spectral fitting results for three of the CXOU J132527.6-430023 spectra are compatible with a $\sim 10 M_{\odot} \mathrm{BH}$ (Figure 6). The best-fit to ObsID 7799 yields a value of $N_{\mathrm{H}}$ that is double that obtained from the other spectra (albeit they are constrained to $90 \%$ uncertainties of $\pm 50 \%$ ). If this is not a real effect, then it could result in an artificial increase in the unabsorbed flux found from fitting. We find a $2 \sigma$ difference between the fits for this ObsID 7799 and a joint fit of the three other bright spectra (Figure 5), indicating that a physical change near the source has occurred. The fitting parameters from ObsID 7799 would be consistent with an $\sim 18 M_{\odot} \mathrm{BH}$; however, it seems very improbable that the compact object gained, and then lost, so much mass during this time.

The spectral fitting process resulted in a best-fit model of an absorbed multicolor disk blackbody (Table 2); the fits to the power-law model lead to extremely steep spectra with $\Gamma>3.7$ and can be ruled out. The absorption column parameter is still above the Galactic value $\left(0.084 \times 10^{22} \mathrm{~cm}^{-2}\right)$; however, judging from the position of the source within Cen A $\left(\alpha=13^{\mathrm{h}} 25^{\mathrm{m}} 27^{\mathrm{S}} .58\right.$, $\delta=-43^{\circ} 00^{\prime} 23^{\prime \prime} .3$, Figure 1$)$, this is not unexpected. The structure of the dust lane is complex, with the source residing in an $\sim 2^{\prime \prime}$ radius quasi-circular region of lower density dust bordered by regions of higher density, which complicates accurately computing the amount of absorption at the source location. All values of $N_{\mathrm{H}}$ from the spectral fitting are consistent with studies of the extinction in the Cen A dust lanes (Schreier et al. 1996), with $0.5<A_{V}<3.0$ indicating an $N_{\mathrm{H}}$ of $(0.11-0.66) \times 10^{22} \mathrm{~cm}^{-2}$ (Güver \& Özel 2009). Analysis of the $K$-band optical depth map presented in Kainulainen et al. (2009) yielded a mean $A_{K}=0.325$, indicating $N_{\mathrm{H}} \sim 0.6 \times 10^{22} \mathrm{~cm}^{-2}$, this would be almost double the absorption column found from three of the spectral fits. The Cen A ULX found by Sivakoff et al. (2008) - outside of the dust lanes - was found to have a similar absorption column to CXOU J132527.6-430023, but only when the source was in the steep power-law state, the column apparently decreasing to consistency with the Galactic value as the thermal component began to dominate the spectrum.

The inner-disk temperatures, $k T_{\text {in }} \sim 0.6 \pm 0.1 \mathrm{keV}$, are softer than the spectra of NS LMXBs, which typically vary between 1 and $2 \mathrm{keV}$. This temperature is also below that of the ULX, which had $k T_{\text {in }} \sim 1 \mathrm{keV}$. Such soft spectra are reminiscent of Galactic $\mathrm{BH}$ transients in the thermal state (McClintock \& Remillard 2005) and are not seen in NS LMXBs of comparable luminosity. The corresponding bolometric luminosities found from the spectral model are $\sim 1.4 \times 10^{38} \mathrm{erg} \mathrm{s}^{-1}$, which equates to $\sim 10 \%$ Eddington for a $10 M_{\odot} \mathrm{BH}$; consistent with known $\mathrm{BH}$ systems in the thermal state. An NS primary emitting at $L_{\text {Edd }}$ seems unlikely based on comparison with Galactic analogues. None of the brighter persistent (Z-track) sources exhibit spectral states as soft, and the only bright NS LMXB transient, XTE J1701-462, exhibited "Atoll-like" behavior (harder spectra) in the 60 days prior to its return to quiescence. Spectral fits of absorbed disk blackbodies to M $31 \mathrm{XBs}$ have also produced $k T_{\text {in }}$ of 0.3-0.6 keV, such as XMMU J004144.7+411110 (Trudolyubov et al. 2006). This source has an estimated unabsorbed luminosity of $3-4 \times 10^{37} \mathrm{erg} \mathrm{s}^{-1}$ for the best-fit spectral model of an 


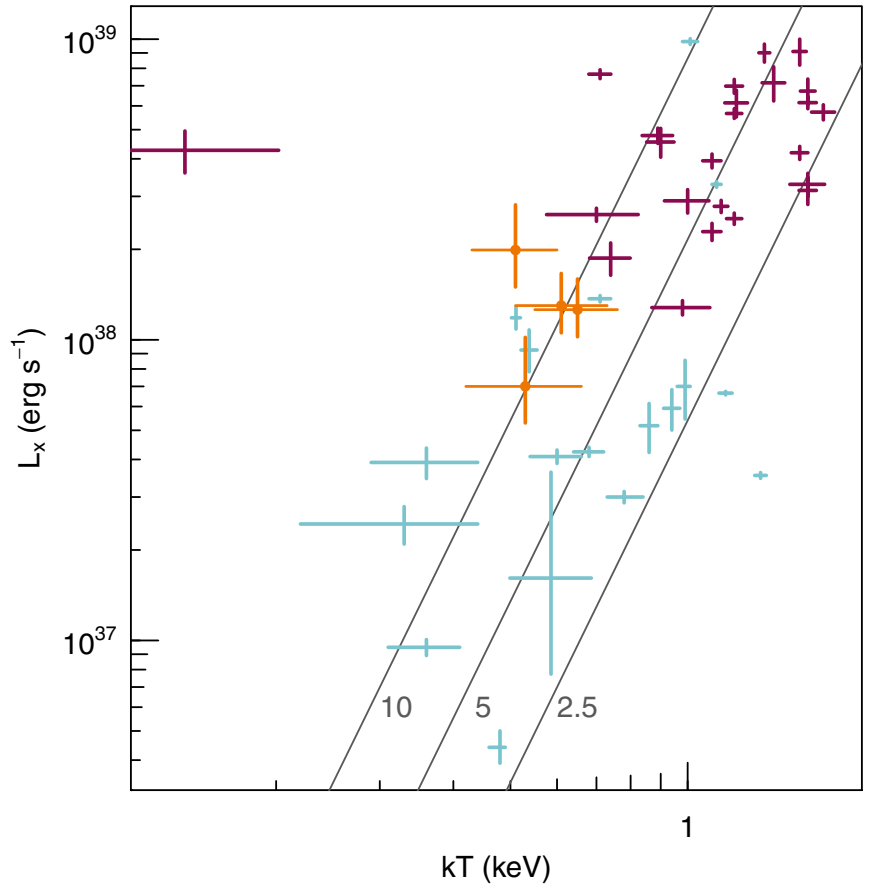

Figure 6. Comparison of $L_{X}-k T_{\text {in }}$ for CXOU J132527.6-430023 (orange with points) with other sources in the thermally dominant state both outside (magenta) and inside (light blue) the Local Group. We make use of the spectral fitting results from sources in NGC 3379 (Brassington et al. 2010) and NGC 4278 (Fabbiano et al. 2010). The Local Group sample is based on spectral fits to Galactic (McClintock \& Remillard 2005; Soria et al. 2011) and M 31 (Trudolyubov et al. 2006; Stiele et al. 2011) sources with $L_{X}$ adjusted to the $0.5-7.0 \mathrm{keV}$ band using Xspec. Lines of constant $\mathrm{BH}$ mass with increasing $k T_{\text {in }}$ for $10 M_{\odot}, 5 M_{\odot}$, and $2.5 M_{\odot} \mathrm{BH}$ are based on the prescription of Gierlinski \& Done (2004) and assumptions of Brassington et al. (2010).

absorbed disk blackbody with $k T_{\text {in }} \sim 0.6-0.8 \mathrm{keV}$. Power-law fits resulted in $\Gamma \sim 2.8-3.3$ and a column density far in excess of Galactic absorption, while the disk blackbody fits resulted in $N_{\mathrm{H}}$ only double that of the Galactic value, very similar behavior to our transient. XMMU J004144.7+411110 also lacks an optical counterpart $\left(m_{v}>21\right)$.

CXOU J132527.6-430023 shows that our study of XB outside the Local Group is starting to probe lower-luminosity BH LMXB with properties more typical of Galactic sources (Figure 6). For the time being, Cen A contains the only population of such objects $\left(10^{37}-10^{38} \mathrm{erg} \mathrm{s}^{-1}\right.$, non-Local Group) that can be subjected to meaningful spectral fitting. In future papers (M. J. Burke et al., in preparation) we will report on detailed spectral analysis of the Cen A XB population of sources with $L_{X}>10^{37} \mathrm{erg} \mathrm{s}^{-1}$ which will, for the first time, allow contrast between the nature of typical LMXBs in our galaxy with analogs from beyond the Local Group.

\section{CONCLUSION}

We believe that the following evidence makes a strong case for CXOU J132527.6-430023 being a transient BH LMXB candidate.

1. Long-term variability. The absorbed flux varies by a factor $>100$ between quiescent and active states on timescales of months. This behavior favors a low-mass companion, as all dynamically confirmed $\mathrm{BH}$ HMXB systems (Cyg X-1 in the Milky Way, LMC X-1 and LMC X-3 in the Large Magellanic Cloud) show persistent X-ray emission (McClintock \& Remillard 2005). This stated, there is now a very strong case for a $\mathrm{BH}$ in HMXBs Cyg X-3 (Shrader et al. 2010) and SS433 (e.g., Blundell et al. 2008), both of which show considerable long-term variability. To date, such variability has been observed in very few NS LMXB systems, while all confirmed BH LMXB, confirmed by direct measurement of the radial velocity and spectral type of the companion, are transients.

2. Spectral fitting results. The best-fit model of an absorbed disk blackbody with a measured inner disk temperature of $\sim 0.6 \mathrm{keV}$ is softer than is typical of NS LMXBs emitting at similar luminosities, and is characteristic of several observed $\mathrm{BH}$ systems in the thermal state. The values of $N_{\mathrm{H}}$ retrieved from fitting are consistent with those derived from source position in $K$-band optical depth maps that were presented in Kainulainen et al. (2009). This is not the case for the absorbed power- law model, which required an extremely steep spectral slope, a much larger value for the absorption column, and achieved a less likely best-fit.

The parameter values of the best-fit absorbed disk blackbody model are typical of an accreting $10 M_{\odot}$ black hole in the thermally dominant state. If indeed this source is an accreting $\mathrm{BH}$, then it is the first confirmed transient non-ULX black hole system in outburst to be identified outside the Local Group.

This work was supported by NASA grant NAS8-03060. M.J.B. thanks the STFC and the University of Birmingham for financial support. R.V. is supported by NWO Vidi grant 016.093.305. G.R.S. acknowledges the support of an NSERC Discovery Grant. We also thank Jeff McClintock, Mike Garcia, Ewan O’Sullivan, Trevor Ponman, \& Alastair Sanderson for useful discussions. Finally, we thank the anonymous referee for their very helpful comments.

\section{REFERENCES}

Arnaud, K. A. 1996, in ASP Conf. Ser. 101, Astronomical Data Analysis Software and Systems V, ed. G. Jacoby \& J. Barnes (San Francisco, CA: ASP), 17

Blundell, K. M., Bowler, M. G., \& Schmidtobreick, L. 2008, ApJ, 678, L47 Brassington, N. J., Fabbiano, G., Blake, S., et al. 2010, ApJ, 725, 1805 Brassington, N. J., Fabbiano, G., Kim, D.-W., et al. 2008, ApJS, 179, 142 Brassington, N. J., Fabbiano, G., Kim, D.-W., et al. 2009, ApJS, 181, 605 Corbel, S., Dubus, G., Tomsick, J. A., et al. 2012, MNRAS, accepted (arXiv:1201.3356v1)

Croston, J. H., Kraft, R. P., Hardcastle, M. J., et al. 2009, MNRAS, 395, 1999

Dickey, J. M., \& Lockman, F. J. 1990, ARA\&A, 28, 215

Dubus, G., Hameury, J.-M., \& Lasota, J.-P. 2001, A\&A, 373, 251

Dubus, G., \& Rutledge, R. E. 2002, MNRAS, 336, 901

Fabbiano, G., Brassington, N. J., Lentati, L., et al. 2010, ApJ, 725, 1824

Ferrarese, L., Mould, J. R., Stetson, P. B., et al. 2007, ApJ, 654, 18

Fender, R. 2005, in Compact Stellar X-ray Sources, Vol. 39, ed. W. H. G. Lewin \& M. van der Klis (Cambridge: Cambridge Univ. Press), 381

Gierliński, M., \& Done, C. 2004, MNRAS, 347, 885

Ghosh, K. K., Finger, M. H., Swartz, D. A., Tennant, A. F., \& Wu, K. 2006, ApJ, 640,459

Goodger, J. L., Hardcastle, M. J., Croston, J. H., et al. 2010, ApJ, 708, 675

Graham, J. A. 1979, ApJ, 232, 60

Güver, T., \& Özel, F. 2009, MNRAS, 400, 2050

Hardcastle, M. J., Kraft, R. P., Sivakoff, G. R., et al. 2007, ApJ, 670, L81

Harris, G. L. H., Gomez, M., Harris, W., et al. 2012, AJ, 143, 84

Harris, G. L. H., Rejkuba, M., \& Harris, W. E. 2010, PASA, 27, 457

Heise, J., \& in't Zand, J. J. M. 2005, in Compact Stellar X-ray Sources, Vol. 157, ed. W. H. G. Lewin \& M. van der Klis (Cambridge: Cambridge Univ. Press)

Homan, J., Kaplan, D. L., van den Berg, M., \& Young, A. J. 2009, ApJ, 692, 73

Homan, J., van der Klis, M., Wijnands, R., et al. 2007, ApJ, 656, 420

Jordán, A., Sivakoff, G. R., McLaughlin, D. E., et al. 2007, ApJ, 671, L117

Kahabka, P., \& van den Heuvel, E. P. J. 1997, ARA\&A, 35, 69

Kainulainen, J. T., Alves, J. F., Beletsky, Y., et al. 2009, A\&A, 502, L5 
Kaur, A., Henze, M., Haberl, F., et al. 2012, A\&A, 538, A49

Kraft, R. P., Hardcastle, M. J., Sivakoff, G. R., et al. 2008, ApJ, 677, L97

Kraft, R. P., Kregenow, J. M., Forman, W. R., Jones, C., \& Murray, S. S. 2001, ApJ, 560, 675

Lin, D., Remillard, R. A., \& Homan, J. 2009, ApJ, 696, 1257

Linares, M., Watts, A., Altamirano, D., et al. 2010, ApJ, 719, L84

Maccarone, T. J., Kundu, A., Zepf, S. E., \& Rhode, K. L. 2007, Nature, 445, 183

Maccarone, T. J., Long, K. S., Knigge, C., Dieball, A., \& Zurek, D. R. 2010, MNRAS, 406, 2087

McClintock, J. E., \& Remillard, R. A. 2005, in Compact Stellar X-ray Sources, Vol. 39, ed. W. H. G. Lewin \& M. van der Klis (Cambridge: Cambridge Univ. Press)

Murdin, P., Jauncey, D. L., Lerche, I., et al. 1980, A\&A, 87, 292

Oosterbroek, T., van der Klis, M., van Paradijs, J., et al. 1997, A\&A, 321, 776

Quillen, A. C., Brookes, M. H., Keene, J., et al. 2006, ApJ, 645, 1092

Remillard, R. A., \& McClintock, J. E. 2006, ARA\&A, 44, 49

Revnivtsev, M. G., \& Gilfanov, M. R. 2006, A\&A, 453, 253

Sanna, A., Méndez, M., Altamirano, D., et al. 2010, MNRAS, 408, 622

Schreier, E. J., Capetti, A., Macchetto, F., Sparks, W. B., \& Ford, H. J. 1996, ApJ, 459,535
Shakura, N. I., \& Sunyaev, R. A. 1973, A\&A, 24, 337

Shih, I. C., Kundu, A., Maccarone, T. J., Zepf, S. E., \& Joseph, T. D. 2010, ApJ, 721,323

Shrader, C. R., Titarchuk, L., \& Shaposhnikov, N. 2010, ApJ, 718, 488

Sivakoff, G. R., Kraft, R. P., Jordán, A., et al. 2008, ApJ, 677, L27

Soria, R., Broderick, J. W., Hao, J., et al. 2011, MNRAS, 415, 410

Stiele, H., Pietsch, W., Haberl, F., et al. 2011, A\&A, 534, A55

Tennant, A. F., Fabian, A. C., \& Shafer, R. A. 1986, MNRAS, 219, 871

Trudolyubov, S., Priedhorsky, W., \& Cordova, F. 2006, ApJ, 645, 27

van den Heuvel, E. P. J., Bhattacharya, D., Nomoto, K., \& Rappaport, S. A. 1992, A\&A, 262, 97

Voss, R., \& Gilfanov, M. 2006, A\&A, 447, 71

Voss, R., Pietsch, W., Haberl, F., et al. 2008, A\&A, 489, 707

Voss, R., Gilfanov, M., Sivakoff, G. R., et al. 2009, ApJ, 701, 471

White, N. E., \& Marshall, F. E. 1984, ApJ, 281, 354

Wijnands, R., \& van der Klis, M. 1999, ApJ, 522, 965

Williams, B. F., Gaetz, T. J., Haberl, F., et al. 2008, ApJ, 680, 1120

Williams, B. F., Naik, S., Garcia, M. R., \& Callanan, P. J. 2006, ApJ, 643, 356

Woodley, K. A., Raychaudhury, S., Kraft, R. P., et al. 2008, ApJ, 682, 199

Worrall, D. M., Birkinshaw, M., Kraft, R. P., et al. 2008, ApJ, 673, L135

Zepf, S. E., Stern, D., Maccarone, T. J., et al. 2008, ApJ, 683, L139 